\title{
Neural responses to dynamic adaptation reveal the dissociation between the processing of the shape of contours and textures
}

\author{
Damien Wright ${ }^{1}$, Benjamin Dering ${ }^{1}$, Jasna Martinovic $^{2} \&$ Elena Gheorghiu $^{1 *}$ \\ ${ }^{1}$ University of Stirling, Department of Psychology, Stirling, FK9 4LA, Scotland, United Kingdom \\ ${ }^{2}$ University of Aberdeen, School of Psychology, Aberdeen, AB24 3FX, Scotland, United Kingdom \\ *Corresponding author: elena.gheorghiu@stir.ac.uk
}

\begin{abstract}
Shape-adaptation studies show that surround textures can inhibit the processing of contours. Using event-related potentials (ERP), we examined the time-course of neural processes involved in contour-shape and texture-shape processing following adaptation to contours and textures. Contours were made of Gabor strings whose orientations were either tangential or orthogonal to the contour path, while textures were made of a series of contours arranged in parallel. We focused on two ERP components $-P 1$, related to low-level visual processes and $N 1$, broadly indicative of mid-level visionand, on ERP difference waves (no-adaptor minus with-adaptor) to isolate the effects of adaptation, which are fundamentally distinct from individual processes driving P1 and N1 components. We found that in the absence of adaptation, the N1 component for contour-tests peaked later and increased in amplitude compared to the $\mathrm{N} 1$ for texture-tests. Following adaptation, the ERP difference wave for contour-tests revealed an early and a late component that were differentially affected by the presence of surround texture, but critically not by its orientation. For texture-tests, the early component was of opposite polarity for contours compared to texture adaptors. From the temporal sequence of ERP modulations, we conclude that texture processing begins before contour processing and encompasses the stages of perceptual processing reflected in both the low-level P1 and the mid-level N1 visionrelated components. Our study provides novel evidence on the nature of separable and temporally distinct texture and contour processing mechanisms, shown in two difference wave components, that highlights the multi-faceted nature of dynamic adaptation to shape when presented in isolation and in context.
\end{abstract}

Keywords: EEG, ERP, adaptation, contours, textures, context, surround, suppression 


\section{Introduction}

Contours and textures play a vital role in object recognition: contours provide information about the shape of objects, whilst textures define surface properties (Biederman, 1987; Marr, 1982). In natural scenes, however, objects rarely occur in isolation but are often surrounded by other objects or set against textured backgrounds. Therefore, understanding how the human visual system segments and recognizes the shape of objects from surrounding textures is crucial for a full understanding of both object recognition and texture perception.

Recent psychophysical and computational studies have shown that textures can modulate the perception of the shape of contours they surround (Gheorghiu \& Kingdom, 2012a, 2012b; Gheorghiu, Kingdom, \& Petkov, 2014; Grigorescu, Petkov, \& Westenberg, 2003, 2004; Kingdom \& Prins, 2009; Li, 1999, 2002; Petkov \& Westenberg, 2003). A novel idea proposed by Gheorghiu, Kingdom and Petkov (2014) is that one functional role of contextual modulation in the visual cortex is to detexturize the image by enhancing the representation of contours at the expense of textures. That is, contours that are part of textures are prevented from acting as contours that define the shapes of objects and thus, are processed by separate mechanisms. Yet, there are also mechanisms dedicated to processing textures, the most popular being the filter-rectify-filter (FRF) models - for a review see Graham (2011) and Landy (2013). These texture-sensitive mechanisms are primarily responsive to changes in textural properties and insensitive to line contours that are not integral to the texture.

Indeed, computational and neurophysiological studies attest to the idea that contour-shapes and texture-shapes are processed by different mechanisms (Grigorescu et al., 2003, 2004; Petkov \& Westenberg, 2003), although whether they are processed in different cortical areas is less clear. It is believed that contour shapes are processed in extra-striate visual areas V4 and IT (Brincat \& Connor, 2004; Ito, Fujita, Tamura, \& Tanaka, 1994; Ito, Tamura, Fujita, \& Tanaka, 1995; Pasupathy \& Connor, 2002), and although some evidence points to textures being processed in striate area V1 (Dumoulin, Dakin, \& Hess, 2008) most fMRI studies suggest that a variety of extra-striate visual areas are involved in processing different aspects and types of textures (Baker, Mortin, Prins, Kingdom, \& Dumoulin, 2006; Cant \& Goodale, 2007; Cavina-Pratesi, Kentridge, Heywood, \& Milner, 2010; Kastner, De Weerd, \& Ungerleider, 2000). In contrast, little is known about the electrophysiological responses involved in texture-shape and contour-shape coding, even though EEG has the potential of measuring the involvement of low-level (striate) vs. mid-level (extra-striate) processes with more temporal precision. In this communication, we use event-related potential (ERP) methods to directly examine the time course of contour-shape and texture-shape processing, and further, investigate the contextual modulation effects on the shape of contours in the absence of, and following, dynamic adaptation.

The existing ERP studies have examined contour and texture processing separately, by focusing either on contour integration and detection from background noise (Machilsen, Novitskiy, Vancleef, \& Wagemans, 2011; Mathes \& Fahle, 2007; Straube, Grimsen, \& Fahle, 2010) or on texture perception (Bach \& Meigen, 1998; Heinrich, Andres, \& Bach, 2007). These studies concentrate on early ERP components - the first positive (P1) and negative (N1) components - which are considered perceptual in nature, while the components that develop later in the time course reflect progressively more cognitive processing. While P1 is largely driven by low-level image content, the N1 is also considered to be a marker of visual discriminative processing and is thought to be generated in extrastriate visual areas (Vogel \& Luck, 2000). Previous ERP studies on contour detection and texture perception used a variety of stimuli that differed in many features (e.g. orientation, spatial frequency, color) and found that the timing of texture segregation, reflected in a negativity occurring at $\sim 110 \mathrm{~ms}$ 
(Heinrich et al., 2007) is earlier than the contour-integration negativity (or CIN), which can be observed at $\sim 150 \mathrm{~ms}$. Thus, evidence suggests that contours and textures produce N1 components of differing latencies (Bach \& Meigen, 1998; Shpaner, Molholm, Forde, \& Foxe, 2013). However, no firm conclusions can be drawn about the timing of these processes as they have not been directly compared within the same study. Therefore, the present study addresses this issue by examining contour-shape and texture-shape processing using comparable stimuli, i.e., textures made from a series of single contours arranged in parallel.

A limited number of studies have examined how context (surround or background) affects ERP responses to contour detection. The presence of a contour embedded in background noise has been shown to elicit a large increase in N1 amplitude compared to background noise only (Mathes \& Fahle, 2007; Shpaner et al., 2013). Only a single study by Machilsen et al (2011) has examined how ERPs were changed by the surround orientation when participants passively viewed closed contours embedded in either uniform or random oriented background elements. These authors found that the presence of the contour increased N1 amplitude (and delayed the peak), but there was no effect on the early P1 component. Conversely, in the absence of a contour, both P1 and N1 component amplitudes were enhanced when the surround orientation was uniform compared to random. Therefore, Machilsen et al (2011) suggest that under passive viewing, the context in which the contour is presented can modulate the ERP response to contour integration. In sum, the abovementioned studies indicate that contour detection in noise is characterized by a pattern of early ERP responses (100-250 ms post-stimulus onset) over occipital/ parietal scalp regions, and more importantly, these ERPs can be modulated by the surround context (uniform vs. random). However, to our knowledge, there are no studies which have examined ERP responses to the shape of contours, when presented in isolation, or embedded in context (or surround texture).

In evaluating ERPs for contour detection from noise, it is worth considering that contour detection/discrimination tasks from background can be achieved by local mechanisms, accomplished by a minimum of neural machinery - for example, the comparison of the orientation of two lines can be achieved by the differences in responses between two orientation-selective neurons (Anzai, Peng, \& Van Essen, 2007; Dobbins, Zucker, \& Cynader, 1987, 1989; Versavel, Orban, \& Lagae, 1990; Wilson, 1985). The neural machinery by which contour-shapes are represented in the brain, as opposed to being detected/discriminated, is better understood through studies of shape appearance (Ben-Shahar \& Zucker, 2004; Gheorghiu \& Kingdom, 2007b, 2008). This is because the perceived shape of a contour is most likely signaled via the population response of neurons tuned to the dimension of interest. One important method to examine shape appearance is the use of adaptation, where the appearance of a stimulus is altered as a result of selective adaptation to a slightly different stimulus. Adaptation paradigms can be successfully combined with the ERP technique as evidenced by ERP adaptation to faces (Eimer, Kiss, \& Nicholas, 2010; Kovacs, Zimmer, Harza, \& Vidnyanszky, 2007) and form and color (Rentzeperis, Nikolaev, Kiper, \& van Leeuwen, 2012). The existing ERP adaptation studies have used static adaptor stimuli and reported that the ERP amplitude is reduced when the adaptor and test stimuli are the same, which is often considered a classic signature of shared processing resources (Grill-Spector, Henson, \& Martin, 2006). However, the use of static adaptors makes it likely that afterimages or adaptation to local information, such as local orientation, might have contributed to the measured effects as was shown to be the case for form/curvature adaptation using static adaptors (Blakemore \& Over, 1974; Stromeyer 3rd \& Riggs, 1974). To avoid these issues, Gheorghiu and colleagues used dynamic adaptor stimuli in which the shape-phase of the contour or texture adaptor was randomly changed every half-second during adaptation (Gheorghiu \& Kingdom, 2006, 2007b; Gheorghiu et al., 2014). Based on studies using static adaptation, we could predict that 
in general, all ERP amplitudes in the present study will be reduced due to the effects of adaptation. However, Rentzeperis et al. (2012) used changing Glass pattern adaptors, and found no P1 or N1 but only late time window $(300-500 \mathrm{~ms})$ adaptation effects. Given that we employ dynamic stimuli that avoid adaptation to local information, we may predict no differences between 'with' and 'without' adaptation conditions in P1 and N1 components, rather, evidence for adaptation effects would likely manifest later in the ERP signal.

Adaptation to shape procedures have provided key evidence that one role of contextual modulation is to de-texturize the image by enhancing the representation of contours at the expense of textures - for a review see Gheorghiu et al (2014). In a shape aftereffect, the perceived shape frequency of a sinusoidal-modulated test contour is altered following adaptation to a contour of a slightly different shape-frequency (Gheorghiu \& Kingdom, 2007b), see Fig.1a. Importantly, the shape aftereffect has been observed not only in contours but also in textures made from a series of sinusoidal-shaped contours arranged in parallel, with both texture-shape and contour-shape aftereffects being of comparable magnitude (Gheorghiu \& Kingdom, 2012a; 2016). Shape aftereffects induced in a single contour-test are reduced when the adaptor consists not of a single contour, but a series of same-orientation contours arranged in parallel to form a texture (Fig. 1b,g), a phenomenon known as 'texture-surround suppression of contour-shape', or TSSCS (Gheorghiu \& Kingdom, 2012b; 2011; Kingdom \& Prins, 2009). However, when the orientations of the surround contours were orthogonal to the path of the central contour (Fig.1c,h), the amount of suppression was reduced, thus indicating that TSSCS is orientation-selective. Thus, TSSCS represents an important neural substrate underlying efficient figure (object) - ground (texture) segregation. Here, we will further examine the neural correlates of TSSCS orientation selectivity.

In this study, we use ERP methods to investigate the neural time-course of processes involved in the adaptation to contour-shape, texture-shape and texture-surround suppression of contour-shape by asking four inter-related questions. First, we ask whether there are differences in the ERP responses to contour-shape and texture-shape in the absence of adaptation. Based on existing literature, we expect that contours and textures will produce differences in P1 and N1 components over posterior scalp regions. Earlier peak latencies for textures would suggest that texture processing occurs earlier in time, which might be a prerequisite for them to be able to prevent contours that are part of textures from acting as contours that define the shapes of objects. Second, we investigate postadaptation differences in ERP responses to contour tests and TSSCS orientation selectivity. For both we predict that dynamic adaptation will result in larger ERP differences in a late time window, i.e., not P1 and N1. Furthermore, the amplitude of the adaptation effect, predicted to occur around 300500ms (see Rentzeperis et al. (2012)), for the same-orientation surround adaptor condition should be similar to the no-adaptor condition, whereas the orthogonal-surround should produce amplitudes comparable to the no-surround (single contour) adaptor. Third, we examine whether texture-shape and contour-shape adaptors produce different post-adaptation ERP responses to texture tests. If we consider the 'uncrossed' adaptor-test conditions (in which the adaptor and test are both the same, either contours or textures) and the 'crossed' conditions (in which the adaptors are textures and the tests are contours, or vice-versa), then a dissociation between the effects measured in the 'crossed' and 'uncrossed' conditions would imply that the texture shape and contour shape are processed by different mechanisms. Finally, we compare ERP responses to two types of contour-shapes (and texture-shapes) made of Gabor strings whose orientations were either tangential (often termed 'snakes') or orthogonal (often termed 'ladders') to the path of the contour (Fig.1f-j). To the best of our knowledge, snakes and ladders stimuli have never been studied with ERPs. Extensive behavioural evidence indicates that these two types of contours are processed by different mechanisms (Bosking, 
Zhang, Schofield, \& Fitzpatrick, 1997; Hess, Hayes, \& Field, 2003; Ledgeway, Hess, \& Geisler, 2005; Li, 1998; May \& Hess, 2007). If this is the case, then we expect to also see a dissociation in our ERP effects, therefore enabling us to refine our understanding of the different processes involved in coding snakes and ladder stimuli. Altogether, these experiments will provide an in-depth, direct comparison of the time-course of neural processes involved in contour-shape, texture-shape, and contextual modulation of the shape of contours in the absence of and following adaptation to snake and ladder contour-shape and texture-shape.

\section{Methods}

\subsection{Participants}

A total of forty-six participants, who were all naive with regard to the experimental aims, took part in this study (23 participants in Experiment1, using snake stimuli and 23 participants in Experiment 2, using the ladder stimuli). All participants (age range: 18-47 years, mean age 21 years, 14 males and 32 female) had normal or corrected-to-normal vision. Participants gave their written informed consent prior to participating in the study and were treated in accordance with the Declaration of Helsinki (2008, version 6). The research protocol was approved by the Research Ethics Committee, University of Stirling, UK.

\subsection{Stimuli - generation and display}

The stimuli were generated using a ViSaGe video-graphics system (Cambridge Research Systems, UK) with a 12-bit contrast resolution and presented on a calibrated, gamma-corrected 20" ViewSonic Graphics Series G225f cathode ray tube (CRT) monitor (ViewSonic, Brea, CA, USA), running at $60 \mathrm{~Hz}$ frame rate and with spatial resolution of $1024 \times 768$ pixels. The mean luminance of the monitor was $65.5 \mathrm{~cd} / \mathrm{m}^{2}$. The experimental setup was placed in a dark, sound attenuated room. Viewing distance was $100 \mathrm{~cm}$.

The adapting stimuli consisted of pairs of sinewave-shaped textures or single contours presented in the center of the monitor, on the mean luminance background at $3.5 \mathrm{deg}$, above and below the fixation marker (see Fig.1a-c and Fig.1f-h). The test stimuli were always pairs of single contours (Fig.1d,e) or textures (Fig.1e,j). The texture adaptors were made of 13 contours and consisted of a central contour flanked by a surround made of a series of contours arranged in parallel. Stimuli were similar to those used by Gheorghiu and Kingdom (2012a, 2012b, 2017, 2019). The adapting stimuli consisted of textures or contours with shape frequency of 0.2 and $0.6 \mathrm{cpd}$, resulting in a geometric mean shape-frequency of $0.35 \mathrm{cpd}$. The mean shape-frequency of the test contour pair was always kept constant at $0.35 \mathrm{cpd}$ and the shape amplitude was fixed at $0.3 \mathrm{deg}$. In all experiments, the shape-frequency of the pair of test-contours was always physically identical (i.e., no physical difference in the shape frequency of the top and bottom test contour) and fixed at $0.35 \mathrm{cpd}$, value which is identical to the geometric mean shape-frequency of the two adaptors. We chose to use pairs of contour/texture adaptors, rather than a single adaptor, because the aftereffect reverses direction as the adaptor shape-frequency switches from being lower to higher than the test shape frequency, implying that aftereffects are bi-directional as demonstrated by Gheorghiu and Kingdom (2007b).

All contours were constructed from strings of odd-symmetric Gabor patches with a spatial bandwidth of 1.75 octaves, a luminance spatial frequency of $5 \mathrm{cpd}$, and a Michelson contrast of $85 \%$. 


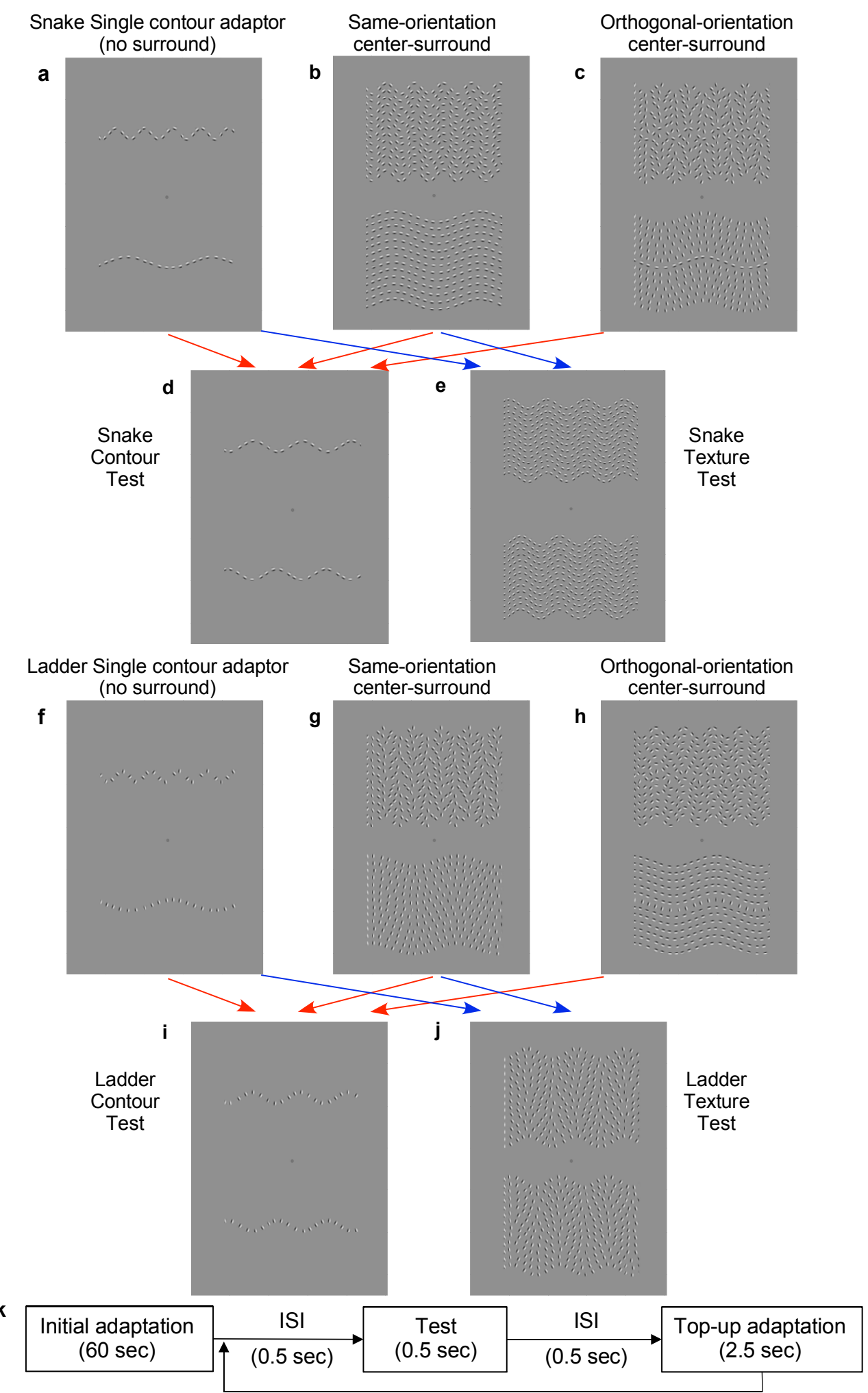

Figure 1. Stimuli and procedure used in Experiment 1 and 2. (a-e) Adaptor and test stimuli used in Experiment 1 (snake contours and textures). (a) Snake single contour adaptor (no surround); (b-c) Texture adaptors made of a snake centralcontour flanked by (b) same-orientation surround i.e., snake surround, and (c) orthogonal-orientation surround i.e., ladder surround. (d) Snake contour test. (e) Snake texture test. (f-j) Adaptor and test stimuli used in Experiment 2 (ladder contours and textures). (f) Ladder single-contour adaptor (i.e., no surround); ( $g$ - $h$ ) Texture adaptors made of a ladder central-contour flanked by (g) same-orientation surround (i.e., ladder surround), and (h) orthogonal-orientation surround (i.e., snake surround). (i) Ladder contour test. (j) Ladder texture test. ( $k$ ) A schematic of the adaptation procedure used in the both experiments. The experiment started with a no-adaptation session (or test only) followed by a typical adaptation session. Each with-adaptation session consisted of an initial adaptation period (60 sec), followed by a repeated test stimulus $(0.5 \mathrm{sec})$ interspersed with top-up adaptation $(2.5 \mathrm{sec})$ periods that were used to reinforce the initial adaptation. An inter-stimulus interval (ISI) of 500ms was inserted before and after each test stimulus. During the ISI, a grey (average luminance) background was presented. 
Gabor patches were positioned along a sinewave-shaped path of the contour and were oriented either tangentially (commonly termed 'snake') or perpendicular (commonly termed 'ladder') to the path of the contour. The center-to-center spacing between adjacent Gabor patches along the contour was randomly selected from within the range $\pm 0.15 \mathrm{deg}$ around a mean of $0.45 \mathrm{deg}$. The stimulus width was $8 \mathrm{deg}$. Due to this, the number of Gabor patches that made up the contours differed by a factor of 1.21 between the high and low shape-frequency adaptor contours, with the former containing 23 Gabor patches and the latter containing 19 Gabor patches. The test contours averaged 20 Gabor patches. We used two types of contours (and textures) stimuli: in Experiment 1, we used snake central-contour adaptor/test and snake texture-tests (Fig.1d,e) and in Experiment 2, the centralcontour adaptor/test and texture-tests were ladders (Fig.1i,j).

To examine center-surround interactions in contour-shape processing, we measure ERPs to pairs of single contour tests (Fig.1d,i) following adaptation to three types of adaptors: central-contour only (i.e., no surround, Fig.1a,f), central contour flanked by same orientation surround (i.e., snake central-contour flanked by snake surround - Fig. 1b; ladder central-contour flanked by ladder surround - Fig.1g), and central contour flanked by orthogonal-orientation surround (i.e., snake central-contour flanked by ladder surround - Fig.1c; ladder central-contour flanked by snake surround - Fig.1h).

Similarly, we examine texture-shape processing by measuring ERPs for texture tests (Fig.1e, j) following adaptation to two types of adaptors: central-contour only (i.e., snake contour - Fig.1a; ladder contour - Fig.1f) and texture (i.e., central and surround contours which have the same orientations: snake textures - Fig.1b; ladder textures - Fig.1g). The test stimuli were pairs of textures (i.e. snake textures - Fig.1e; ladder textures - Fig.1j).

In addition, we also measured ERP responses in contour tests and texture tests in the absence of adaptation (i.e., no-adaptation or baseline condition). For these baseline conditions, the physical shape-frequency of the two test contours (or test textures) was kept constant at $0.35 \mathrm{cpd}$ (i.e. no physical difference in the shape frequency of the top and bottom test contour), value which is identical to the geometric mean of the shape frequency of the adaptor pair: 0.2 and $0.6 \mathrm{cpd}$ ).

\subsection{Procedure - Adaptation}

Each experimental session started with a no-adaptation (or test only) session followed by a typical adaptation session. In the no-adaptor (or baseline) session, we measured the ERP responses to pairs of contour tests (or texture tests) in the absence of the adapting stimulus. Each with-adaptation session consisted of an initial adaptation period of $60 \mathrm{sec}$, followed by a repeated test stimulus of 0.5 sec duration interspersed with top-up adaptation of $2.5 \mathrm{sec}$ that were used to reinforce the initial adaptation. An inter-stimulus interval (ISI) of $500 \mathrm{~ms}$ was inserted before and after each test stimulus. During the ISI, a grey (mean luminance) background was presented. A schematic representation of the test-adapt-test procedure is shown in Fig. 1k. This is a well-established adaptation paradigm used to study many visual adaptation phenomena (Frisby \& Stone, 2010).

During the adaptation period, the shape phase of the sinusoidal-shaped contour was randomly changed every $0.5 \mathrm{sec}$ in order to prevent the formation of after-images and to minimize the effects of local orientation adaptation. The shape-phase of the test contour was also randomly assigned in every test period. The presentation of the test contour was signaled by a tone. Participants were required to maintain fixation on a marker placed between each pair of adaptor and test stimuli for the entire session. On each trial, participants passively viewed the stimuli by fixating between the pairs of adaptor and test stimuli. We used passive viewing rather than a behavioral task to measure aftereffects (by physically changing the shape-frequency of the two test contours until the point of 
subjective equality). This was done because our aim was to compare ERP responses induced by different adaptor stimuli in physically identical test stimuli, i.e., to isolate an adaptation effect in ERPs without the confound of physical changes in the shape frequency of test stimuli. After each adaptation session, participants were given a five minutes break to un-adapt.

For each adaptor and test condition in both experiments, we ran two sessions - one in which the higher shape-frequency adaptor $(0.6 \mathrm{cpd})$ was placed at the top, and the lower shape-frequency adaptor $(0.2 \mathrm{cpd})$ was placed at the bottom, whilst in the other session, this placement order was reversed. During each of these adaptation sessions, a total of 36 test stimuli were presented. Thus, given the two sessions, this resulted in 72 test stimuli per with-adaptor condition. Similarly, the noadaptor (or baseline) condition also contained 72 test stimuli (i.e., 2 sessions x 36 test stimuli).

\subsection{Procedure - EEG recording and analysis}

Electroencephalograms (EEGs) were recorded from the scalp using SynAmps 2 amplifier and Scan 4.5 software (Neuroscan Inc., El Paso TX, USA) at a $1 \mathrm{kHz}$ sampling rate from $64 \mathrm{Ag} / \mathrm{AgCl}$ electrodes positioned according to the extended 10-20 system and using $\mathrm{Cz}$ as the online reference. Four external channels recorded bipolar horizontal and vertical electro-oculograms (EOGs). All electrode recording impedances were kept below $5 \mathrm{~K} \Omega$. The EEG was filtered in real time (on-line) between 0.01 and $200 \mathrm{~Hz}$ and off-line with a low pass $40 \mathrm{~Hz}$ elliptic IIR filter (-144 dB/octave) and down-sampled to $128 \mathrm{~Hz}$ for ease of data analysis.

For each participant, EEG recordings were segmented into epochs ranging from $-100 \mathrm{~ms}$ to $500 \mathrm{~ms}$ after test stimulus onset and averaged according to the experimental condition. Ocular, muscle and other artefacts were identified and removed using Independent Component Analysis, or ICA (Jung et al., 2000). For Experiment 1 (snake stimuli), on average 2.8 components were removed per participant via visual inspection. For Experiment 2 (ladder stimuli), on average 6.5 components were removed per participant. Following ICA, trials exceeding $\pm 100 \mu \mathrm{V}$ in any given epoch were discarded. For Experiment 1, an average of 65 trials were analyzed per condition with between $0-35 \%$ of trials being rejected per participant, whilst in Experiment 2, there was an average of 67 trials per condition with $0-36 \%$ of trials being rejected per participant. Grand-averages for each experiment were calculated after re-referencing individual participant ERPs to the common average reference.

EEG signals are observed via the impact of physiological (i.e., neuronal) activity on the surrounding medium - a possibly localized source produces signals at all electrodes, but the amplitude (and, by proxy, the signal-to-noise ratio of the source's impact) depends on the position of the electrode relative to the source - for in-depth discussion see Maris, (2012). Electrode selection is often performed by selecting a scalp area and time-window based on previous work and then finetuning the selection based on inspection of grand mean data (Luck, 2005). In order to be more objective in our approach, we applied a cluster-based permutation test using the FieldTrip toolbox in Matlab (Oostenveld, Fries, Maris, \& Schoffelen, 2011) to identify the electrodes where the maximal difference between baseline contour and texture responses is observed. We found a significant channel-time cluster (Monte Carlo; $p$-value < 0.05) consisting of 10 posterior electrodes (POZ, PO3, PO4, PO5, PO6, PO7, PO8, OZ, O1, O2). This time window corresponds to the early and late amplitude differences between contours and textures, starting at the onset of the N1 time window, $150 \mathrm{~ms}$, and extending to $383 \mathrm{~ms}$ (see Fig.2 for averaged amplitude differences across channels). Statistical analysis for both Experiment 1 and 2 was performed on the grand average of these electrodes. P1 and N1 were the main components of interest in the analysis. For each individual participant, the peak amplitude for P1 was defined as the highest positive amplitude deflection 
between 70-150 ms, while the peak amplitude for N1 was the largest negativity between 150-230 ms over posterior electrodes. Average amplitude of these peaks was then calculated for each participant by taking the peak value in the corresponding time window, along with the values of the two timepoints (i.e., $15.6 \mathrm{~ms}$ ) either side of the peak.

To examine the effect of adaptation and its time course (i.e., the onset of early and late differences), we also include an analysis of difference waves over the ERP epoch. Difference waves were computed as the difference between the ERP responses corresponding to the contour-tests stimuli for the no-adaptor condition and with-adaptor conditions. These difference waves were computed in a similar manner as the magnitude of shape aftereffects measured in psychophysical adaptation studies, i.e., by taking the differences between no-adaptor and with-adaptor shapefrequency ratios measured in contour tests (or texture tests) at the point of subjective equality (Gheorghiu \& Kingdom, 2012a, 2012b, 2017; Gheorghiu et al., 2014; Gheorghiu, Kingdom, Thai, \& Sampasivam, 2009; Kingdom \& Prins, 2009). Thus, these difference waves over the ERP epoch reflect the effects of adaptation on the neural response.

When examining the difference waves, we found modulations in an early window $70-230 \mathrm{~ms}$, which we refer to as 'the early component', as well as in a late window 250-500 ms, which we term 'the late component'. It is worth noting that ERP difference waves isolate the effects of adaptation and this is fundamentally distinct from individual processes driving P1 and N1 components elicited by test stimuli in a no-adaptation paradigm. However, to pinpoint differential effects of adaptation on sub-processes reflected by the P1 and N1 components, we also compared ERPs responses to test stimuli in the no adaptor (baseline) and with-adaptor conditions. Latency and amplitude differences were again assessed with one-way repeated measures ANOVAs, with factor type of adaptor. The Greenhouse-Geisser correction was applied for all reported ANOVA analyses, and post-hoc tests were performed using paired-samples t-tests, with Bonferroni correction for multiple comparisons. Finally, we show topographic differences corresponding to the difference wave, calculated using the R package eegUtils, version v0.2.0 (Craddock, 2018).

\section{Results}

\subsection{ERP responses to contours and textures in the absence of adaptation}

Figure 2 shows the ERPs for snake (Fig.2a) and ladder (Fig.2b) contour-test (red lines) and texture-test (blue lines) in the absence of adaptation. For snake contours and textures (Fig.2a), P1 occurred $11 \mathrm{~ms}$ earlier for textures than contours $(\mathrm{t}(22)=9.045, \mathrm{p}<0.001)$, with no differences in amplitude $(\mathrm{t}(22)=0.404, \mathrm{p}=0.690)$. The $\mathrm{N} 1$ also occurred $27 \mathrm{~ms}$ earlier for textures than contours $(\mathrm{t}(22)=4.338, \mathrm{p}<0.001)$ and was significantly reduced in amplitude for textures compared to contours $(\mathrm{t}(22)=4.015, \mathrm{p}<0.001)$. Topographic plots indicate a stronger negativity in posterior brain regions for snake contours (Fig.2c) compared to snake textures (Fig.2d).

For ladder contours and textures (Fig.2b), P1 components were of comparable latency ( $\mathrm{t}(22)$ $=1.5, \mathrm{p}=0.148)$. This lack of a difference in P1 latency may result from the larger variability between participants (note the broader bi-modal P1 peak for textures in Fig 2b; however, only 3 individuals showed a bi-modal P1 for ladder-texture tests). However, the amplitude was found to be significantly larger for textures compared to contours $(\mathrm{t}(22)=3.553, \mathrm{p}=0.001)$. As for the $\mathrm{N} 1$ component, this was earlier $(\mathrm{t}(22)=4.222, \mathrm{p}<0.001)$ and less pronounced $(\mathrm{t}(22)=4.045, \mathrm{p}<0.001)$ for textures $(180$ $\mathrm{ms})$ compared to contours (189 ms). Topographic plots for ladder contours (Fig.2e) and textures (Fig.2f) corroborate a stronger negativity in posterior brain regions for contours compared to textures. 
In sum, in the absence of adaptation, contours and textures produce early ERP differences in P1 and N1 for both snake and ladder stimuli, with the observed N1 topographies and amplitude differences suggesting that contours and textures may be processed by different neural mechanisms with different time courses.
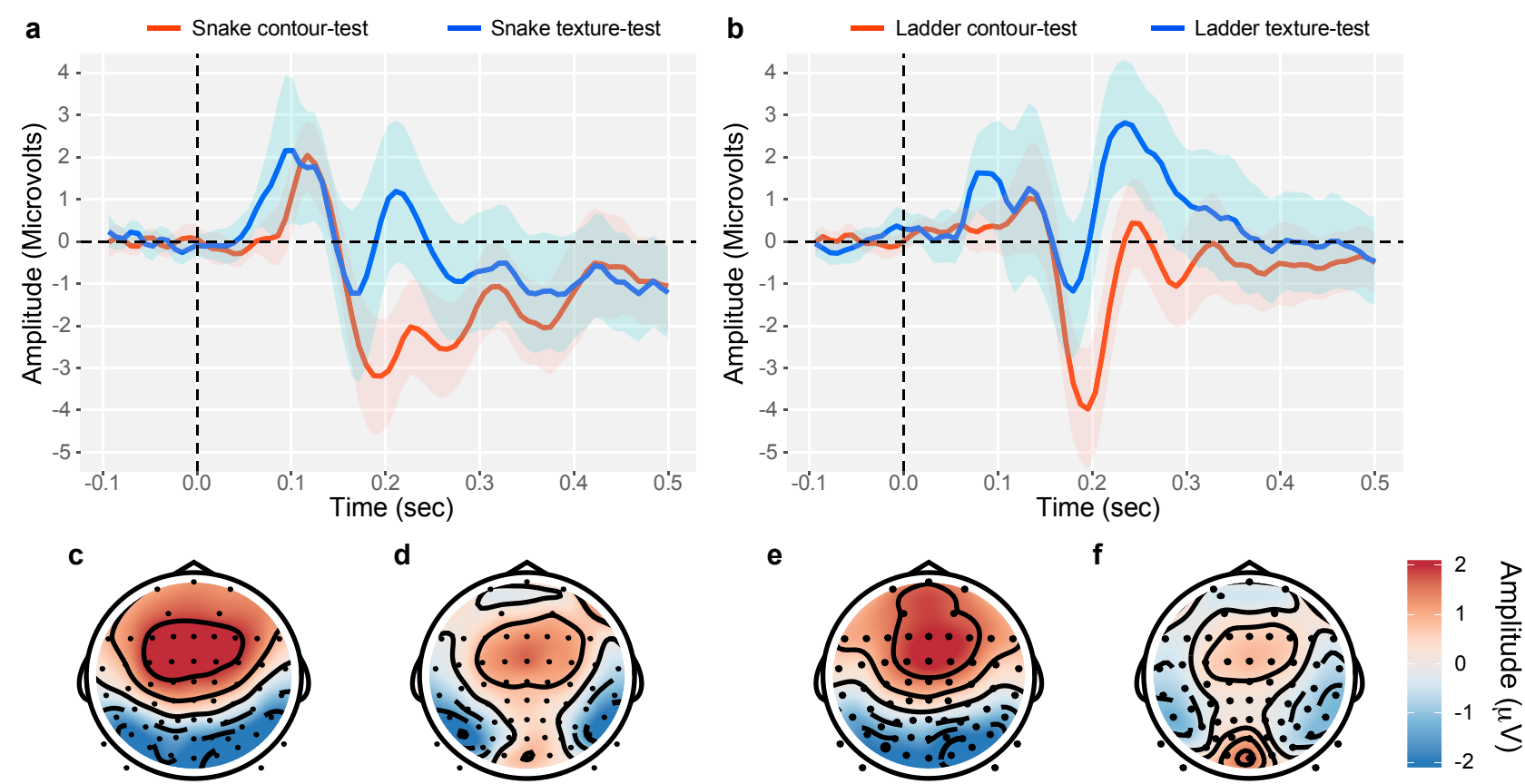

Figure 2. ERPs and topographic plots for contours and textures in the absence of adaptation. Grand average ERPs for (a) snake and (b) ladder contours (red line) and textures (blue line). The colored regions/bands around the ERPs indicate 95\% confidence intervals. Topographic plots corresponding to the N1 time window $(150-230 \mathrm{~ms})$ for (c) snake contours, (d) snake textures, (e) ladder contours and (f) ladder textures.

\subsection{Effect of texture-surround adaptor on the ERP responses to contour tests.}

Figure 3a shows the ERP responses for snake contour-tests before adaptation, i.e. no adaptor condition (red line) and after adaptation to a single central-contour adaptor (i.e. no surround; light green), snake central-contour adaptor flanked by same-orientation (intermediate green) and orthogonal-orientation (dark green) surround. The difference wave between the no adaptor (red line) and with-adaptor (green lines) conditions are shown in Fig.3b. The corresponding ERPs and difference waves for the ladder contour-tests are shown in Fig.3f and 3g, respectively.

For snake contour-tests (Fig.3a), P1 latency was significantly affected by the type of adaptor $\left(\mathrm{F}(3,66)=4.141, \mathrm{p}=0.014, \eta_{p}^{2}=0.158\right)$, with $\mathrm{P} 1$ occurring $10 \mathrm{~ms}$ earlier for orthogonal-orientation surround than the no-surround (or, contour only) adaptor condition $(\mathrm{t}(22)=3.589, \mathrm{p}=0.009)$. There were no other significant latency differences between the different adaptors. Alongside latency, $P 1$ amplitude was also modulated by the type of adaptor $\left(\mathrm{F}(3,66)=5.188, \mathrm{p}=0.006, \eta_{p}^{2}=0.190\right)$, with amplitude being largest for the with-adaptor conditions compared to the no-adaptor condition. Bonferroni corrected multiple comparisons showed that the P1 amplitude for the no surround (t(22) $=4.307, \mathrm{p}=0.017)$ and orthogonal-orientation surround $(\mathrm{t}(22)=3.187, \mathrm{p}=0.025)$ were significantly larger than the no adaptor (or baseline) condition. There were no significant differences between any with-adaptor conditions or between no adaptor and same-orientation surround $(p>0.05)$. As for the 

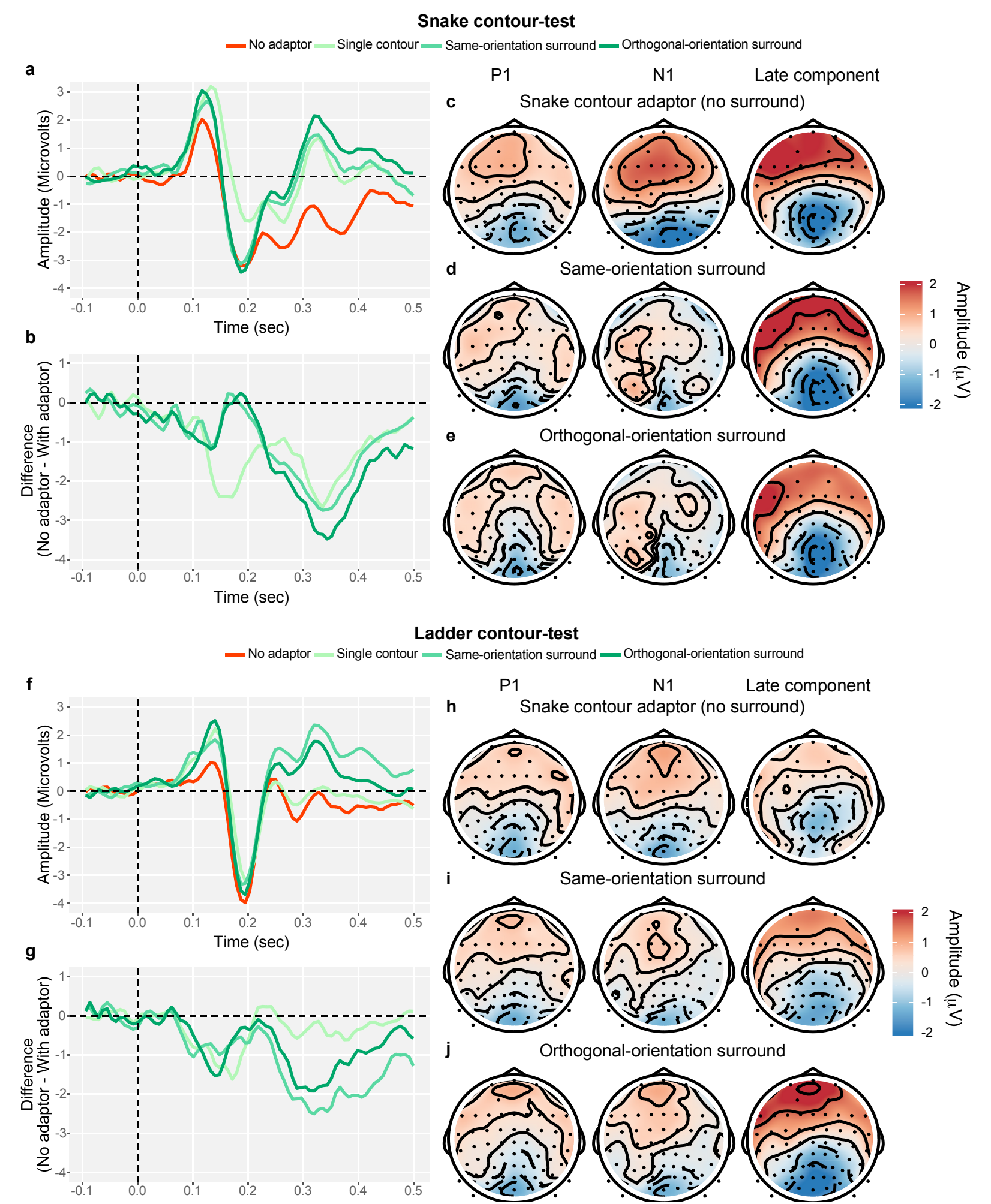

Ladder contour-test
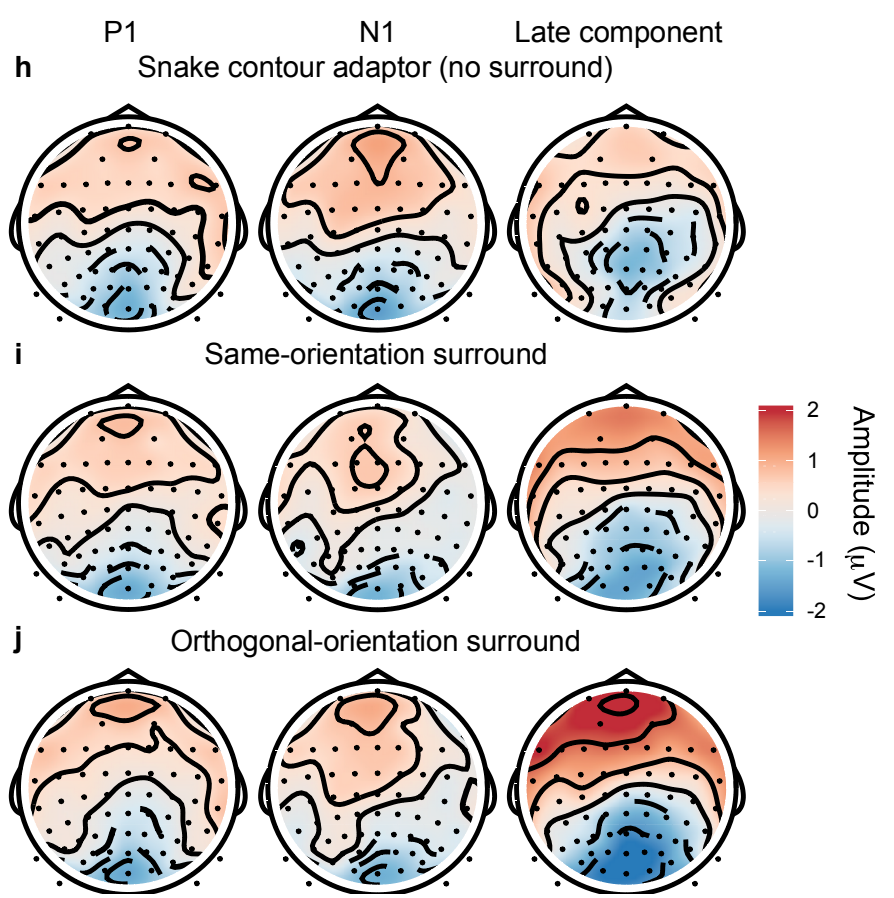

Figure 3. ERPs, difference waves and topographic difference plots for contour tests. (a) Grand average ERPs for snake contour-tests in the absence of adaptation (red line) and following adaptation to single contour (light green) same orientation (intermediate green) and orthogonal-orientation (dark green) center and surround adaptor. (b) Difference wave (no adaptor - with adaptor) for snake contours-tests. (c-j) Topographic difference plots (no adaptor - with adaptor) showing P1 (70-150 ms), N1 (150 - 230 ms), and the late component $(250-500 \mathrm{~ms})$ for (c) single snake-contour adaptor i.e. no surround, (d) orthogonal-orientation surround and (e) same-orientation snake-center and surround. (f) Grand average ERPs for ladder contour-tests in the absence of adaptation (red line) and following adaptation to single contour (light green) same orientation (intermediate green) and orthogonalorientation (dark green) center and surround adaptor. (b) Difference wave (no adaptor - with adaptor) for ladder contours-tests. (c-j) Topographic difference plots (no-adaptor minus with-adaptor) showing P1, N1 and the late component for (c) single ladder-contour adaptor i.e. no surround, (d) orthogonal-orientation surround and (e) same-orientation ladder center and surround. 
N1 component, we found that the type of adaptor also modulated N1 latency $(\mathrm{F}(3,66)=3.799, \mathrm{p}=$ $\left.0.021, \eta_{p}^{2}=0.147\right)$ and amplitude $\left(\mathrm{F}(3,66)=6.051, \mathrm{p}=0.003, \eta_{p}^{2}=0.216\right)$. The $\mathrm{N} 1$ amplitude was found to be reduced for the single contour adaptor compared to the no adaptor condition $(\mathrm{t}(22)=$ 4.365, $\mathrm{p}=0.0015)$. However, all pairwise comparisons on N1 latency showed no significant differences between conditions $(\mathrm{p}>0.05)$.

An analysis of ERP difference waves between no-adaptor and with-adaptor conditions (Fig.3b) indicates the presence of an early (70-230ms) and a late $(250-500 \mathrm{~ms})$ component. To determine if adaptation had an effect for each with-adaptor conditions, we examined whether the ERP difference waves were significantly different from zero. One sample t-tests revealed that in the early time window, only single contour adaptor $(\mathrm{t}(22)=-4.895, \mathrm{p}<0.001)$ was significantly different from zero. For the late time window, single contour or no-surround $(\mathrm{t}(22)=-3.475, \mathrm{p}=0.002)$, sameorientation $(\mathrm{t}(22)=-3.438, \mathrm{p}=0.002)$ and orthogonal-orientation $(\mathrm{t}(22)=-5.322, \mathrm{p}<0.001)$ surround were all significantly different from zero.

We then tested for differences in the magnitude of the ERP difference wave between withadaptor conditions (i.e. no surround vs same-orientation vs orthogonal-orientation surround). The ANOVA showed that the effect of surround adaptor on the amplitude of the early component of the difference wave was significant $\left(\mathrm{F}(2,44)=5.341, \mathrm{p}=0.014, \eta_{p}^{2}=0.195\right)$. Bonferroni corrected multiple comparisons however showed no significant amplitude differences between any of the surround conditions $(\mathrm{p}>0.05)$. As for the late component of the difference wave, there were no significant differences in amplitude between any type of surround adaptor (no surround vs. sameorientation vs. orthogonal-orientation surround; $\mathrm{F}(2,44)=2.528, \mathrm{p}=0.115, \eta_{p}^{2}=0.103$ ). Bonferronicorrected pairwise comparisons showed no significant differences between any conditions $(\mathrm{p}>0.05)$.

Topographic difference plots (Fig.3c-d, reflecting no-adaptor minus with-adaptor conditions) for the P1 window showed that all three types of adaptors show a focal difference over medial occipital/parietal-occipital electrode sites. For the N1 window, the topographic difference plots indicate a similar pattern, with particularly strong differences in medial occipital/parietal-occipital electrode sites for the single contour adaptor (i.e. no surround) condition. As for the late time window, difference topographies were similar for all adaptation conditions, suggesting that the largest differences for adaptation compared to baseline occur in posterior and unilateral frontal electrode locations.

For the ladder contour-test conditions (Fig.3i), P1 amplitude was significantly increased by adaptation $\left(\mathrm{F}(3,66)=6.907, \mathrm{p}<0.001, \eta_{p}^{2}=0.238\right)$, with same-orientation surround $(\mathrm{t}(22)=3.847$, $\mathrm{p}=0.005)$, orthogonal-orientation surround $(\mathrm{t}(22)=3.389, \mathrm{p}=0.015)$ and no-surround adaptor $(\mathrm{t}(22)$ $=3.631, \mathrm{p}=0.009)$ conditions being more pronounced than the no adaptor condition. There were no significant latency differences $(\mathrm{F}(3,66)=1.445, \mathrm{p}=0.246)$ between any of the conditions. As for $N 1$, there were no significant $\mathrm{N} 1$ amplitude differences $(\mathrm{F}(3,66)=1.003, \mathrm{p}=0.397)$ between any conditions. However, we found significant latency differences $\left(\mathrm{F}(3,66)=3.324, \mathrm{p}=0.044, \eta_{p}^{2}=\right.$ $0.131)$ between the conditions, with $\mathrm{N} 1$ occurring later $(194 \mathrm{~ms})$ for the single contour $(\mathrm{t}(23)=3.275$, $\mathrm{p}=0.02)$ and same-orientation surround $(\mathrm{t}(23)=3.117, \mathrm{p}=0.016)$ adaptor conditions $(198 \mathrm{~ms})$ than the no adaptor/ baseline condition (189 ms).

To determine if ERP difference waves for each with-adaptor conditions were different from zero, we used one-sample t-tests. The analysis revealed that in the early time window, similar to snake-contours, only the ladder contour adaptor condition $(\mathrm{t}(22)=-2.741, \mathrm{p}=0.012)$ was significant from zero. For the late time window, only same-orientation surround $(\mathrm{t}(22)=-4.967, \mathrm{p}<0.001)$ was significantly different from zero. 
We then tested for differences in the magnitude of the ERP difference wave between withadaptor conditions (i.e. no surround vs same-orientation vs orthogonal-orientation surround). The ANOVA showed that (i) the early component, which peaked at $\sim 150 \mathrm{~ms}$, was not affected by the type of adaptor (no surround vs same-orientation vs orthogonal-orientation surround; $F(2,44)=0.322$, $p=$ $\left.0.715, \eta_{p}^{2}=0.014\right)$, and (ii) the late component $(250-500 \mathrm{~ms})$ was modulated by the type of adaptor $\left(\mathrm{F}(2,44)=4.032, \mathrm{p}=0.0495, \eta_{p}^{2}=0.154\right)$, with larger amplitude for the same-orientation, followed by the orthogonal-orientation surround and no-surround conditions. Bonferroni corrected multiple comparisons showed a significant larger amplitude in the same-orientation surround than no surround condition $(\mathrm{t}(22)=4.951, \mathrm{p}<0.001)$. There were no significant differences in amplitude between the orthogonal surround and no surround $(\mathrm{t}(22)=0.6408, \mathrm{p}>0.999)$ or same orientation surround $(\mathrm{t}(22)$ $=2.249, \mathrm{p}=0.105)$ conditions.

The topographic difference plots for the P1 window showed similar strength posterior differences for all adaptor types (no surround, same-orientation and orthogonal-orientation surround). This was also the case for the $\mathrm{N} 1$ time window. For the late component, maximal differences for with-adaptor conditions were observed again in posterior occipital electrode sites, with orthogonalsurround adaptor condition producing the strongest difference from the no adaptor/ baseline condition, followed by same-orientation surround and then no-surround adaptor conditions.

In sum, the ERP difference waves for the snake contour-tests indicate that the early component was affected by the presence of a surround-texture adaptor but the effect was agnostic to the orientation of the texture-surround (i.e. same vs. orthogonal-orientation surround), while the late component $(250-500 \mathrm{~ms})$ was not modulated by the type of adaptor. For the ladder contour-test, the opposite was found: while the early component $(70-230 \mathrm{~ms})$ was not affected by the type of adaptor, the late component (250-500 ms) was modulated by the presence of a texture-surround adaptor but the effect was not sensitive to the surround orientation (i.e. same vs. orthogonal-orientation surround).

\subsection{Effect of adaptor type on the ERP responses to texture tests}

Figure 4a shows the ERP responses for snake texture-tests in the absence of adaptation (i.e. no adaptor; blue line) and after adaptation to either single snake-contour (light magenta) and snake textures (dark magenta). The difference wave between the no-adaptor and with-adaptor conditions are shown in Fig. $4 \mathrm{~b}$. The corresponding ERPs and difference waves for the ladder texture-tests are shown in Fig.4e and 4f, respectively.

For snake texture-tests (Fig.4a), there were significant differences in both P1 latency $(\mathrm{F}(2,44)$ $\left.=92.45, \mathrm{p}<0.001, \eta_{p}^{2}=0.808\right)$ and amplitude $\left(\mathrm{F}(2,44)=5.529, \mathrm{p}=0.002, \eta_{p}^{2}=0.201\right)$ between noadaptor and with-adaptor conditions. Latency of the $\mathrm{P} 1$ for contour adaptor peaked at $90 \mathrm{~ms}$, which was $31 \mathrm{~ms}$ earlier compared to the texture adaptor condition $(\mathrm{t}(22)=7.473, \mathrm{p}<0.001)$. The $\mathrm{P} 1$ also peaked $14 \mathrm{~ms}$ earlier for the no adaptor, at $107 \mathrm{~ms}$, compared to the texture adaptor, at $121 \mathrm{~ms},(\mathrm{t}(22)$ $=9.857, \mathrm{p}<0.001)$ condition. There were also significant $\mathrm{P} 1$ amplitude differences depending on the adaptor type, with amplitude being largest for the texture adaptor compared to no adaptor $(\mathrm{t}(22)=$ 2.556, $\mathrm{p}=0.042)$ and contour adaptor $(\mathrm{t}(22)=3.121, \mathrm{p}=0.0095)$ conditions.

As for $N 1$, both latency $\left(\mathrm{F}(2,44)=5.806, \mathrm{p}=0.007, \eta_{p}^{2}=0.209\right)$ and amplitude $(\mathrm{F}(2,44)=$ 41.727, $\left.\mathrm{p}<0.001, \eta_{p}^{2}=0.655\right)$ were influenced by adaptation (i.e., no-adaptor vs. texture adaptor) and by the different type of adaptors (contour vs. texture adaptor). N1 peak latency was found to be $14 \mathrm{~ms}$ earlier for contour than texture adaptor $(\mathrm{t}(22)=3.353, \mathrm{p}=0.005)$, while no adaptor and texture adaptor conditions produced N1 peaks with comparable latency $(\mathrm{t}(22)=2.485, \mathrm{p}=0.063)$. 


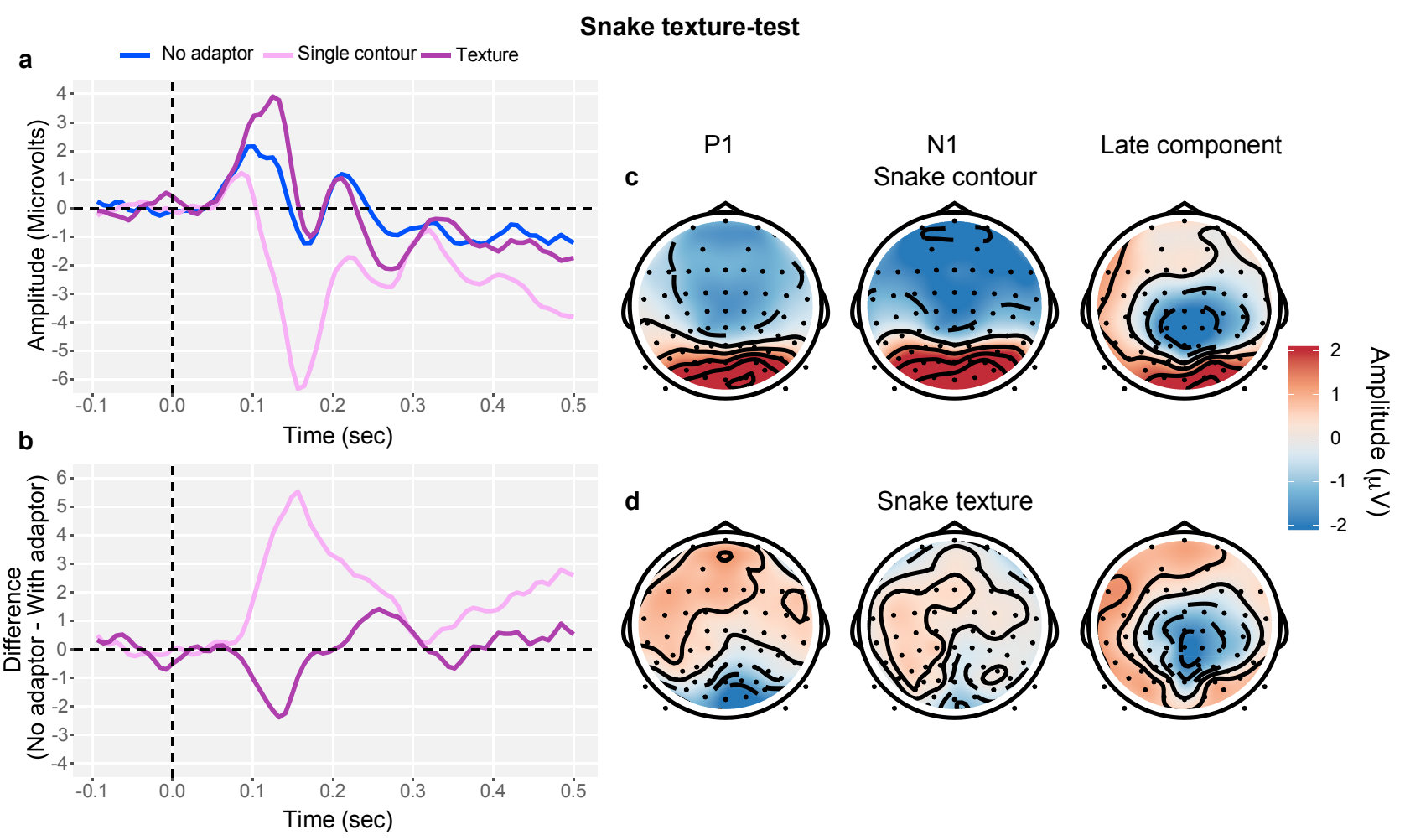

Ladder texture-test
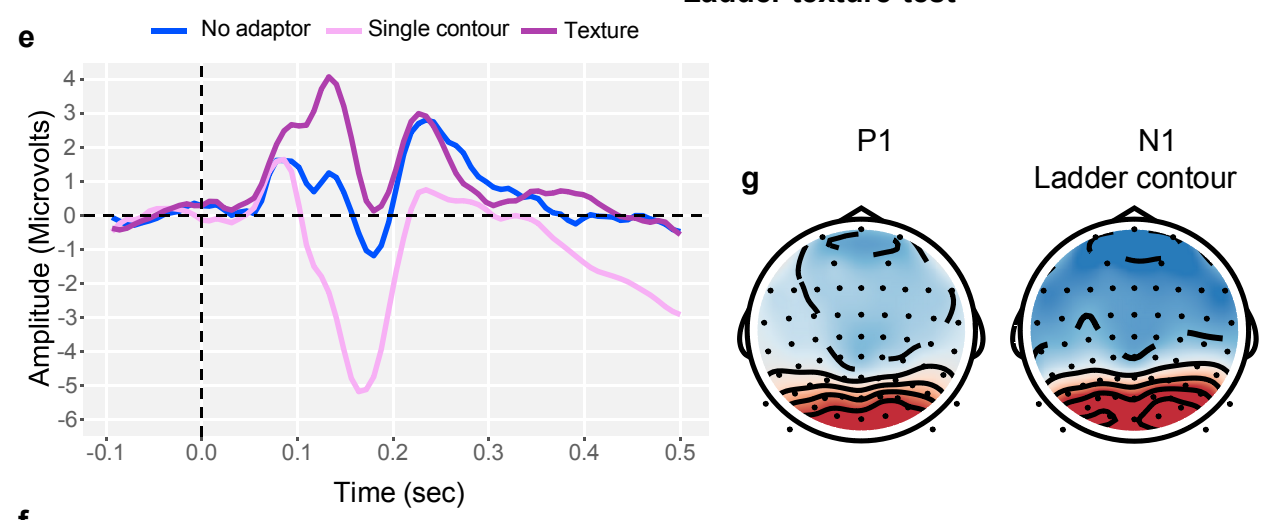

Late component
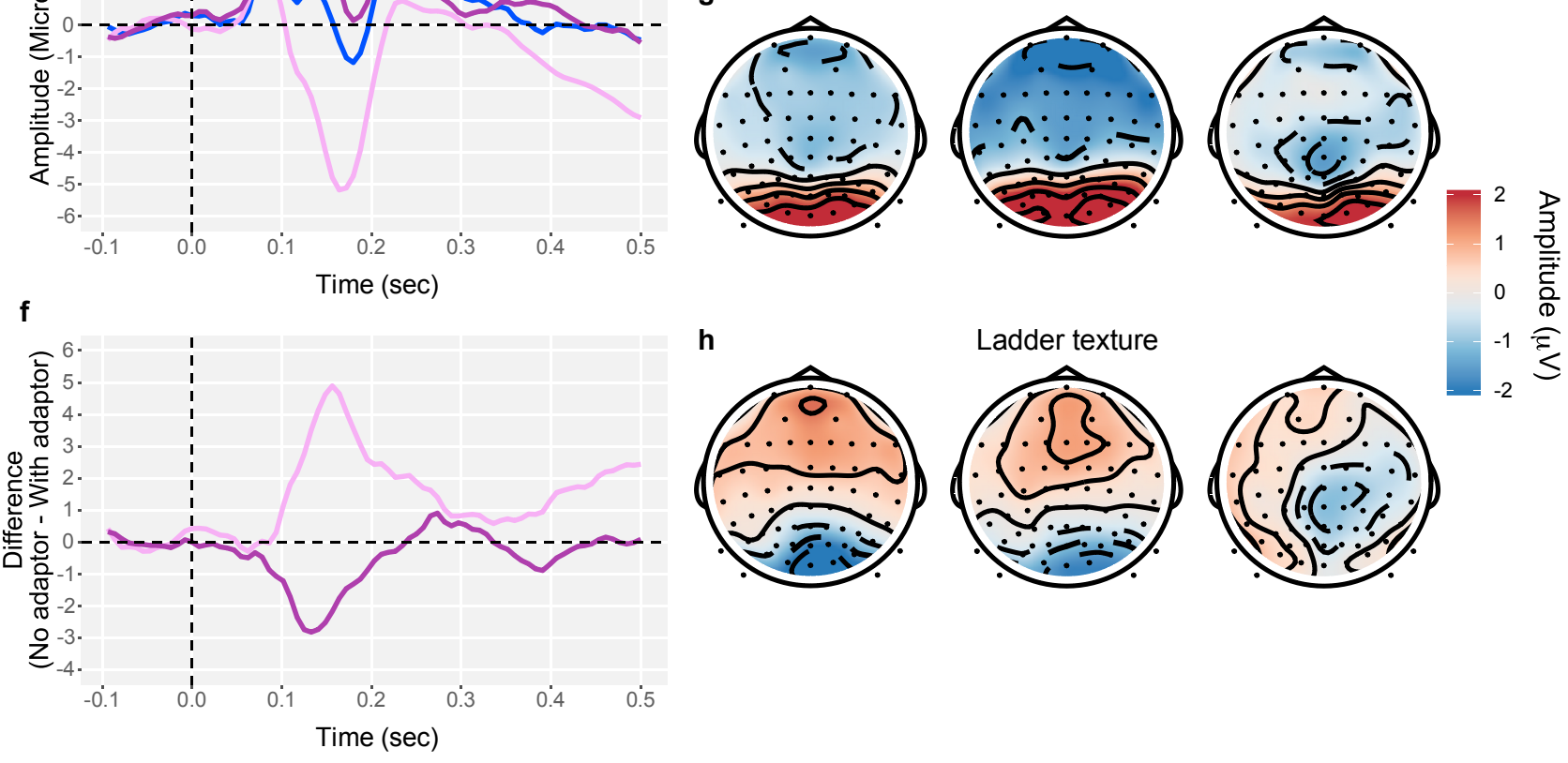

Figure 4. ERPs, difference waves and topographic difference plots for texture-tests. (a) Grand average ERPS for snake texture-tests in the absence of adaptation (blue line) and following adaptation to single snakecontour (light magenta) and snake-texture (dark magenta) adaptor. (b) Difference wave (no adaptor - with adaptor) for snake texture-tests. (c-d) Topographic difference plots (no adaptor-with adaptor) showing P1 $(70-150 \mathrm{~ms}), \mathrm{Nl}(150-230 \mathrm{~ms})$, and the late component $(250-500 \mathrm{~ms})$ for (c) snake-contour and (d) snaketexture adaptors. (e) Grand average ERPs for ladder texture-tests in the absence of adaptation (blue line) and following adaptation to single ladder-contour (light magenta) and ladder-texture (dark magenta) adaptor. $(f)$ Difference wave (no adaptor - with adaptor) for ladder texture-tests. ( $g$ - $h$ ) Topographic difference plots (no adaptor-with adaptor) showing P1, N1 and the late component for (c) ladder-contour and (d) ladder-texture adaptors. 
$\mathrm{N} 1$ amplitude was largest for contour compared to texture adaptor $(\mathrm{t}(22)=7.673, \mathrm{p}<0.001)$ and no adaptor $(\mathrm{t}(22)=8.567, \mathrm{p}<0.001)$ conditions, whilst no adaptor and texture adaptor produces comparable N1 amplitude $(\mathrm{t}(22)=0.951, \mathrm{p}>0.999)$. Consequently, snake contour and texture adaptors induce prominent differences in $\mathrm{N} 1$ for snake texture-tests.

We examined the presence of adaptation by comparing ERP difference waves against zero. This revealed that in the early time window, the single contour adaptor condition was significantly different from zero $(\mathrm{t}(22)=6.598, \mathrm{p}<0.001)$. However, in the late time window, single contour adaptor $(\mathrm{t}(22)=3.253, \mathrm{p}=0.004)$ was significantly different from zero, whilst texture adaptor was $\operatorname{not}(\mathrm{t}(22)=1.057, \mathrm{p}=0.302)$.

We also compared difference waves between all with-adaptor conditions (Fig.4b) and found that: (i) the early component amplitude, peaking at around $150 \mathrm{~ms}$, was of opposite polarity for contours compared to texture adaptors, and (ii) the late component (250-500 ms) was strongly modulated by the type of adaptor (contour vs texture). Both the early $(\mathrm{t}(22)=12.71, \mathrm{p}<0.001)$ and late $(\mathrm{t}(22)=5.308, \mathrm{p}<0.001)$ components showed significant differences between contour and texture adaptors. Topographic difference plots in the P1 window highlight a strong occipital difference to baseline for the contour adaptor condition, yet this difference is weaker, more medial occipital, and flipped in polarity for texture adaptors. Similar to the P1 topography for contours, the N1 topography for the contour adaptor condition showed maximal differences in occipital electrode sites, whereas no strong topographic differences were visible for texture adaptor condition. For the late component, the shape of the topography changes yet remains maximal for contour adaptor condition in posterior occipital electrodes, whilst greatest strength in differences is observed centrally for texture adaptor condition.

For ladder texture-tests (Fig.4e), both $P 1$ latency $\left(\mathrm{F}(2,44)=14.87, \mathrm{p}<0.001, \eta_{p}^{2}=0.403\right.$ ) and amplitude $\left(\mathrm{F}(2,44)=22.53, \mathrm{p}<0.001, \eta_{p}^{2}=0.506\right)$ were significantly affected by adaptor type. $\mathrm{P} 1$ occurred earliest for contour adaptor, at $88 \mathrm{~ms}$, than texture adaptor, at $113 \mathrm{~ms},(\mathrm{t}(23)=5.302, \mathrm{p}$ $<0.001)$ and no adaptor, at $107 \mathrm{~ms},(\mathrm{t}(23)=2.827, \mathrm{p}=0.029)$ conditions. P1 also occurred earlier for no adaptor than texture adaptor $(\mathrm{t}(23)=2.701, \mathrm{p}=0.039)$. P1 amplitude was significantly larger for texture than contour adaptor $(\mathrm{t}(23)=5.332, \mathrm{p}<0.001)$ and no adaptor $(\mathrm{t}(23)=4.882, \mathrm{p}<0.001)$ conditions. The no-adaptor condition also showed a greater $\mathrm{P} 1$ amplitude than contour adaptor $(\mathrm{t}(23)$ $=3.006, \mathrm{p}=0.019)$. The type of adaptor also influenced $N 1$ latency $\left(\mathrm{F}(2,44)=8.459, \mathrm{p}<0.001, \eta_{p}^{2}\right.$ $=0.277)$ and amplitude $\left(\mathrm{F}(2,44)=36.11, \mathrm{p}<0.001, \eta_{p}^{2}=0.621\right)$. The onset of $\mathrm{N} 1$ for ladder texturetests after adaptation to contours, $172 \mathrm{~ms}$, was earlier than for the no-adaptor condition, $180 \mathrm{~ms}$, $(\mathrm{t}(23)$ $=2.826, \mathrm{p}=0.0295)$, and also earlier than texture-adaptor condition, $195 \mathrm{~ms}(\mathrm{t}(23)=3.790, \mathrm{p}=$ 0.003). N1 amplitude was substantially increased for the contour adaptor compared to the no-adaptor $(\mathrm{t}(23)=5.418, \mathrm{p}<0.001)$ and texture-adaptor $(\mathrm{t}(23)=7.376, \mathrm{p}<0.001)$ conditions. $\mathrm{N} 1$ amplitude was more negative for the no adaptor compared with texture- adaptor condition $(\mathrm{t}(23)=2.613, \mathrm{p}=$ 0.047).

To examine whether an adaptation effect was present for ladder texture-tests, ERP difference waves were subjected to one sample t-tests, analyzing amplitude differences against zero. The analysis showed that in the early time window, both texture adaptor $(\mathrm{t}(22)=-4.479, \mathrm{p}<0.001)$ and contour adaptor $(\mathrm{t}(22)=4.981, \mathrm{p}<0.001)$ were significantly different from zero. For the late time window, only single contour adaptor reached significance $(\mathrm{t}(22)=3.836, \mathrm{p}<0.001)$.

An analysis of the ERP difference wave between no-adaptor and with-adaptor conditions for ladder texture-tests (Fig.4f) showed comparable results to those obtained with snake texture-tests. 
The ERP difference waves were strongly modulated by the type of adaptor (contour vs. texture) in both the early $(\mathrm{t}(22)=8.512, \mathrm{p}<0.001)$ and late $(\mathrm{t}(22)=5.418, \mathrm{p}<0.001)$ components.

P1 topographic difference plots for ladder texture-tests were highly similar to snake texturetest difference topographies, following the same pattern. In the case of contour adaptor condition, the $\mathrm{N} 1$ topographic difference was also similar to the snake texture tests, yet the differences between the ladder texture-adaptor and no-adaptor conditions appear maximal over medial occipital electrode sites. Surprisingly, in the late component, adaptation to ladder contours revealed maximal topographic differences in posterior occipital electrode locations, unlike the equivalent snake texture test (compare Fig.4c with Fig.4g). Only weak/small differences were observed for ladder texture adaptation in the late component.

In sum, both snake and ladder texture-tests showed adaptation effects. The ERP difference waves were strongly modulated by the type of adaptor (contour vs. texture) in both the early and late time windows. P1 was enhanced and N1 was reduced when the adaptor and test were both textures.

\section{Discussion}

In this study we examined the time course of processes involved in coding the shape of contours and textures. First, we determined whether there were differences in the ERP responses to contour-shape and texture-shape in the absence of adaptation. Second, we investigated postadaptation differences in ERP responses to contour tests and TSSCS orientation selectivity. Third, we examined whether texture-shape and contour-shape adaptors produced different post-adaptation ERP responses to texture tests. Finally, we compared ERP responses to two types of contour-shapes (and texture-shapes) made of Gabor strings whose orientations were either tangential (often termed 'snakes') or orthogonal (often termed 'ladders') to the path of the contour. One key strength of this study is the use of physically identical test stimuli before and after adaptation to isolate any effects of adaptation. This is considered the gold standard of electrophysiological studies of perception and is known as the Hillyard principle (Pitts \& Martinez, 2014).

We found that in the absence of adaptation, the ERP responses to contours and textures produced negativities from 150-250 ms, with responses to texture tests peaking earlier than contour tests. Following adaptation, P1 and N1 components for contour-tests were both modulated by the presence of adaptors of slightly different shape frequencies. For clarity, P1 and N1 amplitude and latency effects following adaptation are summarized in Table 1 (see Appendix A). We show for the first time that the amplitude of ERP components after dynamic adaptation can increase relative to baseline, no-adaptation conditions. This is in contrast to static adaptation/repetition suppression studies (Eimer et al., 2010; Kovacs et al., 2007). Dynamic adaptation to phase-changing sinewave contours minimizes the effects of low-level, local orientation adaptation. Consequently, attenuation of the P1 in previous static adaptation ERP paradigms is likely to be due to adaptation of low-level feature detectors, rather than any adaptation in mid or high-level vision. Furthermore, since our ERP paradigm largely eliminates low-level contributions to adaptation, we were able to find a dissociation between the effects measured in the 'crossed' (i.e. different adaptor and test) and 'uncrossed' (i.e. same adaptor and test) conditions. ERP difference waves exhibited an early (70-230 ms; covering the $\mathrm{P} 1$ and N1 windows) and a late (250-500 ms) component. For contour-tests, these components were modulated differentially by the presence of texture-surround adaptors (no surround vs. with-surround; see Table 2) but were not sensitive to the surround orientation (same vs orthogonal orientation). For texture-tests, the early component showed opposite polarities for contour and texture adaptors 
conditions, with contour adaptor condition showing a positive potential whilst texture adaptor produced a negativity. The pattern of modulations in these difference waves by (i) adaptation to different type of surround and, (ii) adaptation to contours vs. textures is indicative of different neural mechanisms for the processing of contour-shape and texture-shape, in line with previous psychophysical work. Thus, our ERP study provides an important contribution to the literature by demonstrating that texture processing indeed precedes contour processing and that the locus of interaction between shape of contours and textures can be placed firmly at the stage of mid-level vision, as reflected by the N1 component of the ERP.

A comparison of EEG studies that have examined the neural responses to contour integration (Mathes, Trenner, \& Fahle, 2006) and texture segregation (Bach \& Meigen, 1997) suggests that contour integration and texture segregation produce negativities in the N1 time window, with textures producing an earlier negativity than contours. However, this was not directly shown within the same experiment. Our study is the first to compare the ERP responses to contours and textures using comparable stimuli (i.e. textures made of a series of contours arranged in parallel). We show that in the absence of adaptation, contour and texture tests produced negativities from 150-250 ms, with responses to texture peaking slightly earlier $(\sim 170 \mathrm{~ms})$ than contour tests ( $200 \mathrm{~ms})$. This pattern of results was qualitatively similar for both snake and ladder contours/textures. The earlier response for textures could feed-forward into contour shape-selective neurons in intermediate-to-higher visual areas (Gheorghiu et al., 2014). These findings are in good agreement with previous EEG studies and show that neural markers of texture processing precede those of contour integration.

A few ERP adaptation studies have used the same face adaptor and test stimuli and found that the main effect of adaptation to faces is a reduction in ERP amplitude in the N170 face component (Eimer et al., 2010; Kovacs et al., 2007). Critically, ERP face adaptation studies have used very short adaptation durations $(\leq 5 \mathrm{~s})$ to static face stimuli, in contrast to other visual aftereffect studies which use dynamic adaptors and require longer durations (1 min or longer) of adaptation, e.g. shape aftereffect (Bell, Gheorghiu, \& Kingdom, 2009), tilt aftereffect (Gheorghiu, Bell, \& Kingdom, 2013; Magnussen \& Kurtenbach, 1979), spatial frequency or size aftereffect (Blakemore \& Sutton, 1969), texture aftereffect (Durgin, 1996, 2001). Recent evidence suggests that face adaptation is not restricted to the N170, nor is it category-specific (Feuerriegel, Churches, \& Keage, 2015). Feuerriegel et al., (2015) demonstrate static adaptation can also occur in both P1 and N1 time windows. Furthermore, shorter durations of adaptation, as used for face-adaptation studies, may not be optimal to elicit the onset of the late difference wave component found in the present study (but see Rentzeperis et al., (2012) for Glass pattern adaptation). Given the known sensitivity of P1 and N1 components to visual attention (Hillyard \& Anllo-Vento, 1998), one may think that during the course of an adaption block, attention may decrease in respect to the baseline condition. However, we found that for the no-adaptor condition P1 and N1 amplitude was not significantly increased in comparison to with-adaptor conditions. Importantly, this suggests that attention did not vary within an adaptation block, and thus, changes in ERP component amplitude are due to adaptation effects only.

It is also important to note that several previous ERP adaptation studies used static images as adaptor stimuli and report a reduction in subsequent ERP amplitude measured at the test stimulus (Eimer et al., 2010; Feuerriegel et al., 2015; Kovacs et al., 2007). The use of a static adaptor stimulus can lead to afterimages and adaptation of low-level information, such as orientation and spatial frequency. The present study avoids this problem by dynamically changing the shape phase of the sinusoidal modulated adaptors every $500 \mathrm{~ms}$. This design is well established in shape adaptation studies and has been shown to produce aftereffects in the studied dimension while avoiding adaptation 
of low-level mechanisms, e.g., local orientation, contrast, spatial frequency (Betts, Rainville, \& Wilson, 2008; Frisby \& Stone, 2010; Gheorghiu \& Kingdom, 2007a, 2007b). A single study used a sequence of six Glass pattern adaptors to examine the ERP correlates of adaptation selectivity to color and form (Rentzeperis et al., 2012). These authors report adaptation to both color and form, with no discernable P1 component shown, increased N1 amplitudes observed for color, and a later component (300-500ms) reflecting adaptation to the form of Glass patterns. However, this study could not measure the effect of adaptation due to the lack of a baseline (no-adaptation) condition. We show for the first time that the amplitude of ERP components after dynamic adaptation can increase relative to baseline, no-adaptation conditions. For example, we found that the P1 is enhanced when compared to the baseline for most adaptation conditions, in contrast to static adaptation/repetition suppression studies. This could be explained by the fact that prolonged adaptation to phase-changing sinewave contours minimizes the effects of local orientation adaptation, thus resulting in non-attenuation of the $\mathrm{P} 1$, given that $\mathrm{P} 1$ is known to be sensitive to low level visual information, e.g., contrast (Handy, Soltani, \& Mangun, 2001).

What is the effect of surround-texture adaptors on the ERP responses to snake vs ladder contour-tests? We found that following adaptation, the ERP difference waves highlighted an early (70-230 ms) and a late (250-500 ms) component. For contour tests, these were differently modulated by surround adaptor (no surround vs. same- vs. orthogonal orientation) irrespective of its orientation. For snake contour-tests, only the early component was modulated by the presence of a surround adaptor (no surround vs. with-surround) whereas for ladder contour-tests, the opposite was found: the late component was modulated by the presence of a surround adaptor (no surround vs. withsurround). One may think that the 'good continuity' of the individual central-contour adaptor in the orthogonal-surround adaptor condition might be disrupted by the near elements adjacent to the central-contour adaptor. In our ERP study, we examined the effect of extended texture surround adaptors on the encoding of the shape of a single contour-test (see Fig.1d,i). This is supported by our finding showing that both early and late components of the ERP difference wave for both snake and ladder contour-tests were not modulated by the orientation of the surround adaptor (same vs. orthogonal-orientation surround). This pattern of results can only be due to the different mechanisms involved in processing snakes and ladders (Field, Hayes, \& Hess, 1993; Hess et al., 2003; Ledgeway et al., 2005; May \& Hess, 2007) and not by any disruption to the continuity of contour tests.

Several studies of contour detection/ discrimination from noise texture backgrounds have suggested that snake ( $1^{\text {st }}$ order) contours are segregated from the surrounding elements based on grouping by collinearity, with ladder $\left(2^{\text {nd }}\right.$ order) contour processing involving a $2^{\text {nd }}$ stage of integration (Field et al., 1993; Hess et al., 2003; Ledgeway et al., 2005; May \& Hess, 2007). Studies of contextual modulation of contour shape using adaptation have shown that the magnitude of shape aftereffects measured in contour-tests is dependent on both the size of the surround and the orientation of the surround adaptor (Gheorghiu \& Kingdom, 2012b; Gheorghiu et al., 2014). However, local orientation changes in the texture surround relative to the central contour are unlikely to be evidenced in ERP amplitude fluctuations. This is because both types of surround textures are of similar size, and early visual components of the ERP are arguably more sensitive to global rather than local-changes (e.g., orientation) in stimulus properties. Although our ERP difference waves were agnostic to the orientation of the surround texture (i.e. same vs orthogonal surround), they showed temporal differences in the effects of surrounds on snake and ladder processing (see Table 2).

For both snake and ladder textures, we found that the ERP difference wave for texture tests was modulated by the type of adaptor (contour vs. texture) in both the early and late time windows, with the early window showing a larger component for contour adaptors than for texture adaptor 
conditions. Critically, the direction of these magnitude differences was opposite to one another, suggesting the activation of different neural mechanisms for contour vs texture adaptation. The differences in ERPs we show are in line with extensive psychophysical findings demonstrating that texture-shape aftereffects are reduced when the adaptor is a contour rather than a texture (Gheorghiu \& Kingdom, 2012a, 2012b, 2017, 2019; Gheorghiu et al., 2014; Gheorghiu et al., 2009; Kingdom \& Prins, 2009). These findings complement additional psychophysical and fMRI evidence for the idea that contextual modulation mechanisms highlight contours that are not part of textures - for a review see Gheorghiu et al (2014).

To conclude, our ERP adaptation study provides novel evidence on the nature of separable and temporally distinct texture and contour processing mechanisms. We demonstrated that although previous studies considered both textures and contours to be characterized by a similar N1 negativity, only differing in its latency, there are fundamental differences between texture and contour processing mechanisms. Texture processing starts before contour processing and encompasses both the P1 and the N1 time windows, while contour processing manifests itself within the N1 time window, which is characteristic of mid-level, perceptual organization processes. Our ERP study provides an important contribution to the literature by demonstrating that texture processing indeed precedes contour processing. Furthermore, we showed that texture-surround suppression phenomena are reflected in early (for snake contours) and late (for ladder contours) difference wave components linked to adaptation (see Table 2), but interestingly not by surround orientation. Finally, we demonstrate that in contrast to short-duration static adaptation/ repetition suppression studies, our prolonged dynamic adaptation can uncover different stages of visual processing. It may be interesting for future work to investigate whether adaptation duration influences the presence and/or magnitude of early or late difference wave components linked to adaptation. Our study provides a point of reference for using dynamic adaptation over repetition suppression to study mid and high-level vision processes, without confounds from low-level feature adaptation.

\section{Appendix A}

Table 1. Statistically significant effects and their directions in P1 and N1 time windows for the no-adaptor or baseline condition (B), single contour adaptor or no surround condition $(C)$, contour adaptor flanked by orthogonal-orientation $(O)$ and same-orientation $(S)$ surround. For the texture-test experiment (Experiment 2), the contour adaptor flanked by same-orientation surround is simply referred to as texture adaptor (T).

\begin{tabular}{|r|c|c|c|c|}
\hline & \multicolumn{2}{|c|}{ P1 } & \multicolumn{2}{c|}{ N1 } \\
\hline Contour test & Latency & Amplitude & Latency & Amplitude \\
\hline Snake & $\mathrm{O}<\mathrm{C}$ & $\mathrm{C} \& \mathrm{O}>\mathrm{B}$ & $\mathrm{C}<\mathrm{B}$ \\
\hline Ladder & $\mathrm{C} \& \mathrm{O} \& \mathrm{~S}>\mathrm{B}$ & $\mathrm{B}<\mathrm{C} \& \mathrm{~S}$ & \\
\hline Texture test & $\mathrm{C} \& \mathrm{~B}<\mathrm{T}$ & $\mathrm{T}>\mathrm{B} \& \mathrm{C}$ & $\mathrm{C}<\mathrm{T}$ & $\mathrm{C}>\mathrm{T} \& \mathrm{~B}$ \\
\hline Snake & $\mathrm{C}<\mathrm{B}<\mathrm{T}$ & $\mathrm{T}>\mathrm{B}>\mathrm{C}$ & $\mathrm{C}<\mathrm{T}$ & $\mathrm{C}>\mathrm{B}>\mathrm{T}$ \\
\hline Ladder &
\end{tabular}


Table 2. Statistically significant effects of texture-surround adaptor on the early and late components of the ERP difference wave for snake and ladder contour-tests (Experiment 1). These components were modulated differentially by the presence of texture-surround adaptors (no surround vs. with-surround), but not by the surround orientation (same vs. orthogonal orientation).

\begin{tabular}{|r|c|c|}
\hline & \multicolumn{2}{|c|}{ Effect of texture-surround adaptor on the ERP difference wave } \\
\hline Contour test & Early component $(70-230 \mathrm{~ms})$ & Late component $(250-500 \mathrm{~ms})$ \\
\hline Snake & Yes & No \\
\hline Ladder & No & Yes \\
\hline
\end{tabular}

\section{Supplementary Information}

All data, analyses, stimuli and code are available online at: http://hdl.handle.net/11667/139

No part of the study procedures was pre-registered prior to the research being conducted.

No part of the study analyses was pre-registered prior to the research being.

We report how we determined our sample size, all data exclusions, all inclusion/exclusion criteria, whether inclusion/exclusion criteria were established prior to data analysis, all manipulations, and all measures in the study.

\section{Acknowledgements}

This research was supported by a Leverhulme Trust grant (RPG-2016-056) awarded to Elena Gheorghiu (PI) and Jasna Martinovic (co-PI). The C code used to generate the contours, written in conjunction with routines from the VISAGE graphics library (Cambridge Research System) was modified from code originally written by Frederick A. A. Kingdom. We would like to thank Frederick Kingdom for helping with the development of the original $\mathrm{C}$ code.

\section{Authors Contributions}

Conceived and designed the experiments: EG, JM, DW, BD. Performed the experiments: DW. Analyzed data: DW. Wrote the paper: EG, DW, BD, JM.

\section{Competing Interests}

The authors declare no competing interests.

\section{References}

Anzai, A., Peng, X., \& Van Essen, D. C. (2007). Neurons in monkey visual area V2 encode combinations of orientations. Nat Neurosci, 10(10), 1313-1321. doi: nn1975 [pii] $10.1038 / \mathrm{nn} 1975$

Bach, M., \& Meigen, T. (1997). Similar electrophysiological correlates of texture segregation induced by luminance, orientation, motion and stereo. Vision Res, 37(11), 1409-1414. 
Bach, M., \& Meigen, T. (1998). Electrophysiological correlates of human texture segregation, an overview. Doc Ophthalmol, 95(3-4), 335-347.

Baker, C. L., Mortin, C. L., Prins, N., Kingdom, F. A. A., \& Dumoulin, S. (2006). Visual cortex responses to different texture-defined boundaries: An fMRI study. Journal of Vision, 6(6), 209.

Bell, J., Gheorghiu, E., \& Kingdom, F. A. (2009). Orientation tuning of curvature adaptation reveals both curvature-polarity-selective and non-selective mechanisms. $J$ Vis, 9(12), 3 1-11. doi: $10.1167 / 9.12 .3$

Ben-Shahar, O., \& Zucker, S. W. (2004). Sensitivity to curvatures in orientation-based texture segmentation. Vision Res, 44(3), 257-277. doi: S0042698903006151 [pii]

Betts, L., Rainville, S., \& Wilson, H. (2008). Adaptation to radial frequency patterns in the lateral occipital cortex. Journal of Vision, 8(6), 723-723.

Biederman, I. (1987). Recognition-by-components: a theory of human image understanding. Psychol Rev, 94(2), 115-147.

Blakemore, C., \& Over, R. (1974). Curvature detectors in human vision? Perception, 3(1), 3-7.

Blakemore, C., \& Sutton, P. (1969). Size adaptation: a new aftereffect. Science, 166(3902), 245247.

Bosking, W. H., Zhang, Y., Schofield, B., \& Fitzpatrick, D. (1997). Orientation selectivity and the arrangement of horizontal connections in tree shrew striate cortex. J Neurosci, 17(6), 21122127.

Brincat, S. L., \& Connor, C. E. (2004). Underlying principles of visual shape selectivity in posterior inferotemporal cortex. Nature Neuroscience, 7(8), 880-886. doi: 10.1038/nn1278

Cant, J. S., \& Goodale, M. A. (2007). Attention to form or surface properties modulates different regions of human occipitotemporal cortex. Cereb Cortex, 17(3), 713-731. doi: bhk022 [pii] 10.1093/cercor/bhk022

Cavina-Pratesi, C., Kentridge, R. W., Heywood, C. A., \& Milner, A. D. (2010). Separate processing of texture and form in the ventral stream: evidence from FMRI and visual agnosia. Cereb Cortex, 20(2), 433-446. doi: bhp111 [pii] 10.1093/cercor/bhp111

Craddock, M. (2018). craddm/eegUtils: eegUtils (Version v0.2.0). Zenodo, doi.org/10.5281/zenodo.1292902.

Dobbins, A., Zucker, S. W., \& Cynader, M. S. (1987). Endstopped neurons in the visual cortex as a substrate for calculating curvature. Nature, 329(6138), 438-441. doi: 10.1038/329438a0

Dobbins, A., Zucker, S. W., \& Cynader, M. S. (1989). Endstopping and curvature. Vision Res, 29(10), 1371-1387.

Dumoulin, S. O., Dakin, S. C., \& Hess, R. F. (2008). Sparsely distributed contours dominate extrastriate responses to complex scenes. Neuroimage, 42(2), 890-901. doi: S10538119(08)00592-2 [pii] 10.1016/j.neuroimage.2008.04.266

Durgin, F. H. (1996). Visual aftereffect of texture density contigent on color of frame. Percept Psychophys, 58(2), 207-223.

Durgin, F. H. (2001). Texture contrast aftereffects are monocular; texture density aftereffects are binocular. Vision Res, 41(20), 2619-2630. doi: S0042-6989(01)00121-3 [pii]

Eimer, M., Kiss, M., \& Nicholas, S. (2010). Response profile of the face-sensitive N170 component: a rapid adaptation study. Cereb Cortex, 20(10), 2442-2452. doi: bhp312 [pii] 10.1093/cercor/bhp312

Feuerriegel, D., Churches, O. F., \& Keage, H. A. (2015). Is neural adaptation of the N170 categoryspecific? Effects of adaptor stimulus duration and interstimulus interval. Int $J$ Psychophysiol, 96(1), 8-15. doi: 10.1016/j.ijpsycho.2015.02.030

Field, D. J., Hayes, A., \& Hess, R. F. (1993). Contour integration by the human visual system: evidence for a local "association field". Vision Res, 33(2), 173-193.

Frisby, J. P., \& Stone, J. V. (2010). Seeing aftereffects: the psychologist's microelectrode. (Chapter 4). In: Seeing: The computational approach to biological vision., (Second edition). MIT Press., pp. 75-110. 
Gheorghiu, E., Bell, J., \& Kingdom, F. A. (2013). Line orientation adaptation: local or global? PLoS One, 8(8), e73307. doi: 10.1371/journal.pone.0073307

Gheorghiu, E., \& Kingdom, F. A. (2006). Luminance-contrast properties of contour-shape processing revealed through the shape-frequency after-effect. Vision Res, 46(21), 36033615. doi: S0042-6989(06)00230-6 [pii] 10.1016/j.visres.2006.04.021

Gheorghiu, E., \& Kingdom, F. A. (2007a). Chromatic tuning of contour-shape mechanisms revealed through the shape-frequency and shape-amplitude after-effects. Vision Res, 47(14), 1935-1949. doi: S0042-6989(07)00123-X [pii] 10.1016/j.visres.2007.03.010

Gheorghiu, E., \& Kingdom, F. A. (2007b). The spatial feature underlying the shape-frequency and shape-amplitude after-effects. Vision Res, 47(6), 834-844. doi: S0042-6989(06)00523-2 [pii] $10.1016 /$ j.visres.2006.11.023

Gheorghiu, E., \& Kingdom, F. A. (2008). Spatial properties of curvature-encoding mechanisms revealed through the shape-frequency and shape-amplitude after-effects. Vision Res, 48(9), 1107-1124. doi: S0042-6989(08)00098-9 [pii] 10.1016/j.visres.2008.02.002

Gheorghiu, E., \& Kingdom, F. A. (2012a). Chromatic properties of texture-shape and of texturesurround suppression of contour-shape mechanisms. J Vis, 12(6), 16. doi: 12.6 .16 [pii] $10.1167 / 12.6 .16$

Gheorghiu, E., \& Kingdom, F. A. (2012b). Local and global components of texture-surround suppression of contour-shape coding. $J$ Vis, 12(6), 20. doi: 12.6.20 [pii] 10.1167/12.6.20

Gheorghiu, E., \& Kingdom, F. A. (2017). Dynamics of contextual modulation of perceived shape in human vision. Sci Rep, 7, 43274. doi: 10.1038/srep43274

Gheorghiu, E., \& Kingdom, F. A. (2019). Luminance-contrast properties of texture-shape and texture-surround suppression of contour shape. Journal of Vision, 19, 1-14.

Gheorghiu, E., Kingdom, F. A., \& Petkov, N. (2014). Contextual modulation as de-texturizer. Vision Res, 104, 12-23. doi: S0042-6989(14)00199-0 [pii] 10.1016/j.visres.2014.08.013

Gheorghiu, E., Kingdom, F. A., Thai, M. T., \& Sampasivam, L. (2009). Binocular properties of curvature-encoding mechanisms revealed through two shape after-effects. Vision Res, 49(14), 1765-1774. doi: S0042-6989(09)00151-5 [pii] 10.1016/j.visres.2009.04.010

Gheorghiu, E., \& Kingdom, F. A. A. (2011). Spatial-properties of texture-surround suppression of contour-shape coding. Journal of Vision, 11(11), 1038.

Gheorghiu, E., \& Kingdom, F. A. A. (2016). Luminance-contrast properties of texture-shape and of texture-surround suppression of contour-shape. Journal of Vision, 16(12), 245.

Graham, N. V. (2011). Beyond multiple pattern analyzers modeled as linear filters (as classical V1 simple cells): useful additions of the last 25 years. Vision Res, 51(13), 1397-1430. doi: S0042-6989(11)00047-2 [pii] 10.1016/j.visres.2011.02.007

Grigorescu, C., Petkov, N., \& Westenberg, M. A. (2003). Contour detection based on nonclassical receptive field inhibition. IEEE Trans Image Process, 12(7), 729-739. doi: 10.1109/TIP.2003.814250

Grigorescu, C., Petkov, N., \& Westenberg, M. A. (2004). Contour and boundary detection improved by surround suppression of texture edges. Image and Vision Computing, 22(8), 609-622. doi: Doi 10.1016/J.Imavis.2003.12.004

Grill-Spector, K., Henson, R., \& Martin, A. (2006). Repetition and the brain: neural models of stimulus-specific effects. Trends Cogn Sci, 10(1), 14-23. doi: 10.1016/j.tics.2005.11.006

Handy, T. C., Soltani, M., \& Mangun, G. R. (2001). Perceptual load and visuocortical processing: event-related potentials reveal sensory-level selection. Psychol Sci, 12(3), 213-218. doi: $10.1111 / 1467-9280.00338$

Heinrich, S. P., Andres, M., \& Bach, M. (2007). Attention and visual texture segregation. J Vis, 7(6), 6. doi: 10.1167/7.6.6

Hess, R. F., Hayes, A., \& Field, D. J. (2003). Contour integration and cortical processing. J Physiol Paris, 97(2-3), 105-119.

Hillyard, S. A., \& Anllo-Vento, L. (1998). Event-related brain potentials in the study of visual selective attention. Proc Natl Acad Sci U S A, 95(3), 781-787. doi: 10.1073/pnas.95.3.781 
Ito, M., Fujita, I., Tamura, H., \& Tanaka, K. (1994). Processing of contrast polarity of visual images in inferotemporal cortex of the macaque monkey. Cereb Cortex, 4(5), 499-508.

Ito, M., Tamura, H., Fujita, I., \& Tanaka, K. (1995). Size and Position Invariance of Neuronal Responses in Monkey Inferotemporal Cortex. Journal of Neurophysiology, 73(1), 218-226.

Jung, T. P., Makeig, S., Westerfield, M., Townsend, J., Courchesne, E., \& Sejnowski, T. J. (2000). Removal of eye activity artifacts from visual event-related potentials in normal and clinical subjects. Clin Neurophysiol, 111(10), 1745-1758.

Kastner, S., De Weerd, P., \& Ungerleider, L. G. (2000). Texture segregation in the human visual cortex: A functional MRI study. J Neurophysiol, 83(4), 2453-2457.

Kingdom, F. A., \& Prins, N. (2009). Texture-surround suppression of contour-shape coding in human vision. Neuroreport, 20(1), 5-8. doi: 10.1097/WNR.0b013e32831578ca

Kovacs, G., Zimmer, M., Harza, I., \& Vidnyanszky, Z. (2007). Adaptation duration affects the spatial selectivity of facial aftereffects. Vision Res, 47(25), 3141-3149. doi: $10.1016 /$ j.visres.2007.08.019

Landy, M. S. (2013). Texture analysis and perception. In J. S. Werner \& L. M. Chalupa (Eds.), The New Visual Neurosciences (Ch. 45, pp. 639-652). Cambridge, Mass.: MIT Press.

Ledgeway, T., Hess, R. F., \& Geisler, W. S. (2005). Grouping local orientation and direction signals to extract spatial contours: empirical tests of "association field" models of contour integration. Vision Res, 45(19), 2511-2522.

Li, Z. (1998). A neural model of contour integration in the primary visual cortex. Neural Comput, 10(4), 903-940.

Li, Z. (1999). Visual segmentation by contextual influences via intra-cortical interactions in the primary visual cortex. Network, 10(2), 187-212.

Li, Z. (2002). A saliency map in primary visual cortex. Trends Cogn Sci, 6(1), 9-16. doi: S1364661300018179 [pii]

Luck, S. J. (2005). An introduction to the event-related potential technique. Cambridge, MA: The MIT Press.

Machilsen, B., Novitskiy, N., Vancleef, K., \& Wagemans, J. (2011). Context modulates the ERP signature of contour integration. PLoS One, 6(9), e25151. doi:

10.1371/journal.pone.0025151

Magnussen, S., \& Kurtenbach, W. (1979). A test for contrast-polarity selectivity in the tilt aftereffect. Perception, 8(5), 523-528.

Maris, E. (2012). Statistical testing in electrophysiological studies. Psychophysiology, 49(4), 549565. doi: 10.1111/j.1469-8986.2011.01320.x

Marr, D. (1982). Vision: A computational investigation into the human representation and processing of visual information. San Francisco: W. H.Freeman., ch.3, pp. 215-239.

Mathes, B., \& Fahle, M. (2007). The electrophysiological correlate of contour integration is similar for color and luminance mechanisms. Psychophysiology, 44(2), 305-322. doi: 10.1111/j.1469-8986.2007.00501.x

Mathes, B., Trenner, D., \& Fahle, M. (2006). The electrophysiological correlate of contour integration is modulated by task demands. Brain Res, 1114(1), 98-112. doi: 10.1016/j.brainres.2006.07.068

May, K. A., \& Hess, R. F. (2007). Dynamics of snakes and ladders. J Vis, 7(12), 13 11-19. doi: $10.1167 / 7.12 .13$

Oostenveld, R., Fries, P., Maris, E., \& Schoffelen, J. M. (2011). FieldTrip: Open source software for advanced analysis of MEG, EEG, and invasive electrophysiological data. Comput Intell Neurosci, 2011, 156869. doi: 10.1155/2011/156869

Pasupathy, A., \& Connor, C. E. (2002). Population coding of shape in area V4. Nat Neurosci, 5(12), 1332-1338. doi: $10.1038 / \mathrm{nn} 972$

Petkov, N., \& Westenberg, M. A. (2003). Suppression of contour perception by band-limited noise and its relation to nonclassical receptive field inhibition. Biol Cybern, 88(3), 236-246. doi: $10.1007 / \mathrm{s} 00422-002-0378-2$ 
Pitts, M., \& Martinez, A. (2014). Contour integration: sensory, perceptual and attention-based ERP components. (Chapter 14). in: Cognitive Electrophysiology of Attention: Signals of the Mind. Edited by George R. Mangun. Imprint Academic Press.

Rentzeperis, I., Nikolaev, A. R., Kiper, D. C., \& van Leeuwen, C. (2012). Relationship between neural response and adaptation selectivity to form and color: an ERP study. Front Hum Neurosci, 6, 89. doi: 10.3389/fnhum.2012.00089

Shpaner, M., Molholm, S., Forde, E., \& Foxe, J. J. (2013). Disambiguating the roles of area V1 and the lateral occipital complex (LOC) in contour integration. Neuroimage, 69, 146-156. doi: 10.1016/j.neuroimage.2012.11.023

Straube, S., Grimsen, C., \& Fahle, M. (2010). Electrophysiological correlates of figure-ground segregation directly reflect perceptual saliency. Vision Res, 50(5), 509-521. doi: S00426989(09)00572-0 [pii] 10.1016/j.visres.2009.12.013

Stromeyer 3rd, C. F., \& Riggs, L. A. (1974). Curvature detectors in human vision? . Science, 14(184(142)), 1199-1201.

Versavel, M., Orban, G. A., \& Lagae, L. (1990). Responses of visual cortical neurons to curved stimuli and chevrons. Vision Res, 30(2), 235-248. doi: 10.1016/0042-6989(90)90039-n

Vogel, E. K., \& Luck, S. J. (2000). The visual N1 component as an index of a discrimination process. Psychophysiology, 37(2), 190-203.

Wilson, H. R. (1985). Discrimination of contour curvature: data and theory. J Opt Soc Am A, 2(7), 1191-1199. 